\title{
The stroboscopic human vision
}

\author{
Maarten A. Bouman \\ Helmholtz Instituut, Utrecht University \\ The Netherlands \\ 1 september 2010
}

\begin{abstract}
When the frequency of seeing light from a pair of point flashes is beyond the probability summation of the frequencies of the separate flashes, the surplus is due to the successful interaction of subliminal responses from the different flashes. Experiments with different distances and different periods of the pair show that successful interaction occurs when in each of two successive time-quanta of 0.04 seconds and in each of two adjacent distinct receptor groups at least one subliminal receptor response occurs. An autonomous source produces the time-quanta. It serves the time processing of the central nervous system and of the motor systems. Possibly, action potentials from the purkinje cells of the myocardium play a role. Hyper acuity in direction and in depth, flicker fusion, perceptual rivalry and other phenomena follow from the quantized spatiotemporal signal processing.
\end{abstract}

\section{Introduction}

In the dark-adapted retina, any receptor responds to single quantum absorption. Such a response not suffices for the perception of light. If it did, the threshold energy for perception would not -as it does- depend on duration and size of the stimulus. Already in 1921, LeComte du Nouilly (LeComte du Nouilly 1921) established this crucial fact for rods. Later a flow of circumstantial evidence became available for the conclusion that the same applies for cones (Bouman 1969).

What configuration in time and space of rod or cone responses does the perception of light really need? A simple and effective method for finding the answer to this question is the application to the retina of configurations of probes of very small and very short flashes from an incandescent light source. The latter emits a rigorously constant average number of light quanta per unit time and viewing angle of a spatiotemporal Poisson distribution (Bouman and Brink 1952, Brink and Bouman 1954). The key data are measurements of the probability for seeing light for a single probe $w$ and the probabilities for seeing any light from pairs of identical probes $W(t, d)$ for different mutual distance $t$ and time $d$. When the members of a pair are far apart in time and/or in space 
or when they occur in the different eyes, only probability summation occurs $W$ $=1-(1-w)^{2}$. By experience, light stimulation makes the dark-adapted eye less sensitive for further stimulation (Bouman 1969). Consequently, one may expect that quantum absorptions in the first flash of the pair decrease the effectiveness of further stimulation. However, in all experiments $W$ is never below the value for mere probability summation. Consequently, the excitation of the retina by the first one member not obstructs the excitation by the other member. Therefore, the members of a successful pair of quantum absorptions probably excite distinct different groups of receptors in mutually independent different periods.

Experimental results

When in the experiments, values for $W(t, d)$ beyond $W$ occur; they reveal the successful interactions of subliminal excitations from different probes. This is irrespective of how the perception of light for the single probes separately comes about. For the efficient application of the method, adjustment of the intensity of the single probe with $w \sim 30 \%$ is most appropriate. $W(t, d)$ reaches usually values up to almost $\mathbf{8 0} \%$. This largest value $\mathrm{W}$ is for those distances or periods for which successful interaction always occurs. The definition for the proportion of successful interactions in dependence on mutual period t respectively mutual distance $d$ of the pairs reads $0 \leq \mathbf{f}(\mathbf{t}, \mathbf{d})=\frac{W(\mathbf{t}, \mathbf{d})-W}{\mathbf{W}-W} \leq \mathbf{1}$.

Figure 1

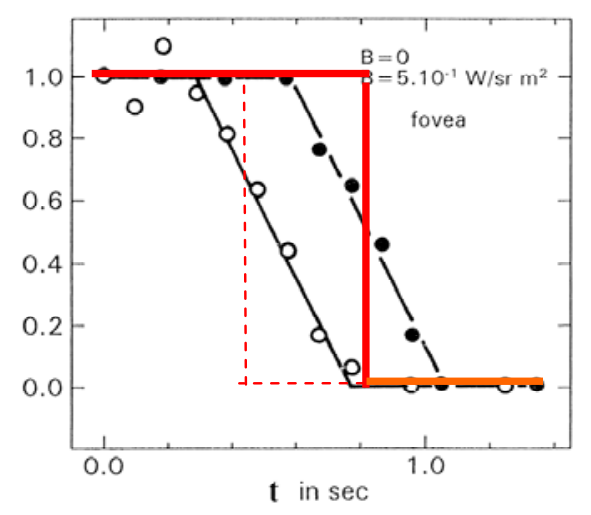


Figure 1 shows in the red curves the probability of successful interaction of two subliminal responses in the fovea as a function of the time $t$ between them, for light of narrow spectral bandwidth around $500 \mathrm{~nm}$. The full drawn red curve applies for in the dark, the dashed curve for intense background. The black curves represent $f(t, d)$ in the dark $(B=0$, dots) and against an intense background $\left(B=5.10^{-1} \mathrm{~W} / \mathrm{sr} \mathrm{m}^{2}\right.$, open circles).

There are three remarkable aspects around these dependences on $t$.

Firstly, the full-drawn black curves of figure 1 are valid for any location on the retina and for any spectral energy distribution of the light. Evidently, the curves are expressions of a basic mechanism for the time processing of receptor responses generally. Either the actual responses of the receptors themselves regulate this mechanism or an autonomous mechanism samples stroboscopic and synchronously all the receptive elements of the system by distinct successive periods. Because for the results of figure 1 the retinal location and kind of stimulated receptors do not matter, the latter possibility is the more likely one.

Secondly, the parts of the curves for $0<f(t, d)<1$ are linear, parallel and covers under all conditions the same range of about 0.042 seconds. This suggests that just the dependence with time of average intensity in the single probe determines this range. The probes were 0.01 seconds in duration with rise and fall time of less than one millisecond. The longest and the shortest possible time between quantum absorptions of different and not overlapping probes of a pair are $t-(0.01+0.001)$ seconds and $t+(0.01+0.001)$ seconds. In the case of autonomous stroboscopic sampling, their difference of 0.022 seconds is operative at the start and finish of a period. Therefore, the transition range in figure 1 should cover at least about 0.044 seconds. Comparison with the actual range of 0.042 seconds leaves strictly no time for the transition from the one to the following sampling period. With reference to an experimental limit of accuracy, the transition lasts less than a few tenths of one millisecond. This implies that the ranges are an almost exact representation of the total average number of quantum absorptions on a relative scale in distinct periods of 0.08 
and 0.04 seconds of both probes together. Thus, subliminal receptor responses can interact successfully when they occur within a distinct period of $\mathbf{0 . 0 8 0}$ seconds in the dark and half this duration of 0.040 seconds against an intense background. The 0.08 seconds period is just the succession of two periods of 0.040 seconds. In the dark, the two subliminal responses should occur each in one of two successive periods of 0.040 seconds. The actual periods will be subject to a statistical spread. This should not- and indeed it does not- affect the linear transitive course of the experimental average curve. These experiments with pairs of very short probes are crucial for understanding of this time processing mechanism of the system.

Thirdly, under light adapted conditions already a single subliminal response should need the interaction of several one-quantum absorptions within $\mathbf{0 . 0 4 0}$ seconds. For the successful interaction with such a subliminal response, adequate nerve signals from the background are continuously available. This availability increases with increasing intensity of the background. Thus, with increasing intensities of the background the transitive parts of the curves in figure 1 move gradually from the line for black dots to the one for the open circles.

Figure 2

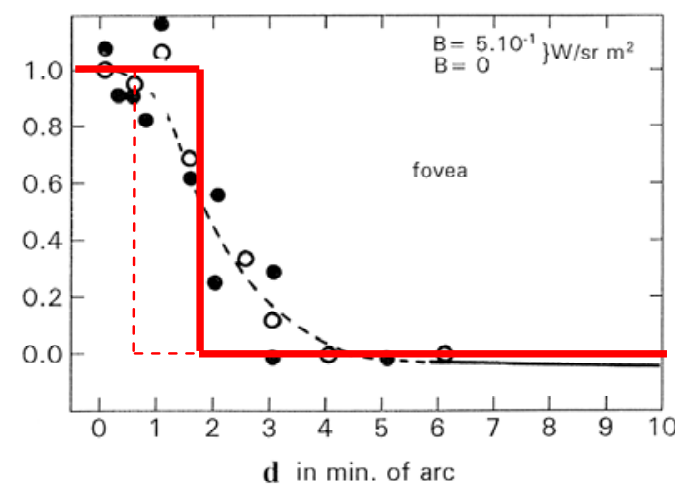

Figure 3

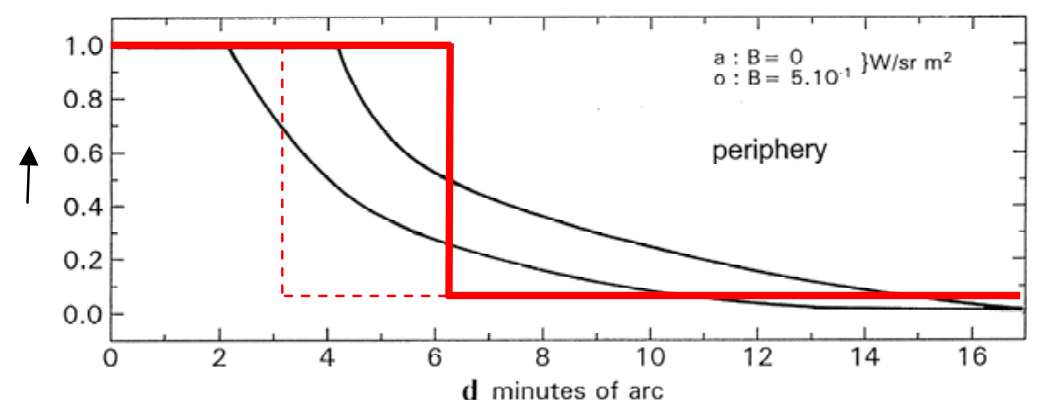

Figure 2 shows for the fovea for $500 \mathrm{~nm}$ light, the results of $f(t, d)$ as a function of $d$ in the dark (black open circles, $B=0$ ) and against background (black dots, $B=5 \cdot 10^{-1} \mathrm{~W} / \mathrm{sr} \mathrm{m}^{2}$ ) The black dashed curve applies for any color of the light and intensity of the background, 
inclusive $B=0$. The red lines are for the probability of successful interaction of subliminal receptor responses in the dark (full drawn) and against intense background (dashed).

Figure 3 shows $f(t, d)$ in the full drawn black curves for seven degrees nasal from the fovea for $500 \mathrm{~nm}$ light, in the $\operatorname{dark}(B=0)$ and against intense background $\left(B=5.10^{-1} \mathrm{~W} / \mathrm{sr} \mathrm{m}^{2}\right)$. The red curves give the probability for successful interaction of subliminal responses in dependence of their distance in the retina, in the dark (full drawn) and against intense background (dashed).

The curve relates to the spatial processing mechanism of subliminal responses. Because of the convergence from the total number of receptors of about $10^{8}$ to the number of fibers in the nervous opticus of about $10^{6}$, likely the receptors situate in distinct groups. In the fovea, for the eye optics the half-width of the optical spread function of an ideal point source is about 0.6 minutes of arc (Vos, Walraven, van Meeteren, 1976). This spread is due to stray light, geometrical optical aberrations and diffraction for the artificial pupil of $\mathbf{2} \mathbf{~ m m}$ diameter. The geometrical optical diameter of the probes is one arc minute. The largest distance between quantum absorptions in different probes of a pair is about $d+(1+0.6)$. The smallest distance for non-overlapping probes is $d$ $(1+0.6)(m i n u t e s$ of arc. Their difference is 3.2 minutes of arc. The transition range $0<f(t, d)<1$ in figure 2 show rather exactly this value. Then there would be no space left for the transition from the one to the neighboring distinct receptor group except the space between adjacent receptors. Because the diameter of a single foveal cone is about $\mathbf{0 . 8}$ minute of arc, in analogy with the facts for the time processing, the distinct single group would contain just one cone. Consequently, the successful interaction of subliminal responses from different probes could stem from single one-quantum absorptions in each of the members of a distinct pair of identical cones. Under light-adapted conditions, a receptor certainly needs several one-quantum absorptions for the deliverance of a response. This need cannot appear with a shift to smaller distances in the experimental results of figure 2. The optical limit of the two probes method does not allow the observation of such decrease. Moreover, 
considerations below show that multifold absorption of quanta in a single receptor does not contribute to the threshold mechanism for perception of mere light. Figure 3 shows the results for 7 degrees nasal from the fovea for $500 \mathrm{~nm}$ light. The transitive parts of the curves as a function of $\mathrm{d}$ for both in the dark as well as against intense background subtends about 8 minutes of arc. The half-width value of the ideal point spread function is at this location a bit larger than at the fovea. An estimate of 1 minute of arc is appropriate. The difference in largest and smallest distances between responding receptors from both probes are about four minutes of arc. The transitive parts in the curves in figure 3 for $0<f(t, d)<1$ cover just about eight minutes of arc. Thus, similarly to the facts for the time processing there is hardly any space left for the transition from the one to the next distinct group of receptors whose responses can successfully interact. In the dark, the interactive distance is 6 minutes of arc and against intense background almost precisely half this value of three minutes of arc. The course of the curves in their transitive parts of figure 3 represent on relative scale the average total number of receptor responses within the distinct distances of 6 and 3 minutes of arc of both probes together. In the peripheral retina, an eccentricity dependent number of rods accompany each single cone. The area of about 3 arc minutes diameter contains at 7 degrees eccentricity around 15 rods and 1 cone and the area of 6 minutes of arc 60 rods and about 4 cones. Against the background, for a subliminal response more than one receptor of a group of receptors should each absorb an increasing number of light quanta. Subliminal signals by receptor responses from the background can successfully interact with subliminal responses from any of the two probes. This way the background contributes to the detection of the presentation of the probes. Therefore, the transitive part of the curves moves with increasing background intensity gradually from the course for the dark to the course for intense background. The conformity of their courses points also to the existence of distinct distances 
and thus distinct neighboring groups of receptors for interaction between subliminal responses in the peripheral retina.

\section{Discussion}

From earlier experiments, rather extended and advanced proposals about interaction between receptor responses are already available. These stems from psychophysical experiments of thresholds for light and color under variation of different spatiotemporal parameters, inclusive moving point sources.(Bouman 1965). In the dark for foveal vision, the area as well as the period for summation of subliminal receptor responses for the perception of light is twice as large as for the perception of color of the light (Walraven 1962, Bouman 1969, 2006). Further earlier experiments suggest that in the dark, light is perceived when in each of a pair of successive distinct periods of 0.04 seconds in each one of a distinct twin pair of groups of receptors at least one quantum is absorbed. The perceived light is in color when at least two quanta are absorbed in a single receptor in one of the two periods of a successive pair of time-quanta of 0.04 seconds. When such occurs in an $R$ cone, the subject sees red, when in a $\mathrm{G}$ cone green. Such absorption in 0.04 seconds in a single rod turns the perceived light into definite white. (Bouman and Walraven 1972, Bouman and Scholten 1976). However, only a twofold quantum absorption in a single cone or rod does not suffice for perception of light or color. Such absorption just adds a colored appearance to the actual perception of light. The existence of these twin pairs of receptor groups and this pairing of successive distinct periods for light and color perception stem the proposals of the present study. Normally, two $R$ pairs and one G pair situate along the three diagonals of a single hexagon. There is a satisfactorily confinement between the results of the present and the earlier study (Bouman 2006). 
The analysis of these earlier results is of straightforward statistical physics nature (Bouman 2006). Altogether, the data lead to rather definite conclusions. For the detection threshold, utmost deterministic conditions hold. In the conscious perception, digital mechanisms of single specific quantum absorption have decisive effect for the appearance of light, color, movement, and edge. This statement is rather exclusive in relation to the present state of the art in cognitive neurosciences. So far, contemporary neural imaging studies reach at results that are still more global.

\section{Conclusions}

Therefore, it is relevant to recapitulate some other well-known phenomena that stem the fairness of the present conclusions.

First, there is the surprising distinctness of interactive distances and periods. The spatial distinctness could near the size of open area of less than 10 arc seconds between adjacent receptors. The temporal distinctness rather nears the rise time of action potentials in the nerve system. This implies that accuracy for discrimination whether a particular receptor response occurs in one or the other of two successive periods or of neighboring receptors or receptor groups is respectively less than half a millisecond or ten arc seconds. There are in vision perceptual phenomena that express such accuracy. For foveal vision, vernier acuity in nonius reading and in binocular vision, the disparity of the two retinal images at the threshold of depth perception both show values that near the $\mathbf{1 0}$ arc seconds. The latter also implies that the registration of the mosaics of receptor groups in the two eyes should occur locally with at least the selfsame accuracy of 10 arc seconds.

In binocular vision, a sunglass of variable optical density before one eye induces an adjustable average time lag around less than a millisecond between the receptor responses of the two retinal images (Mueller 1954). Look now freely at a moving pendulum. An extra illusory adjustable depth movement of 
the pendulum appears. This is because synchronous receptor responses stem from disparate images. This so-called Pulfrich pendulum illusion demonstrates that also the accuracy for perception of synchrony between the two retinal images nears half a millisecond.

Effectively, these pairings embody a perfect vehicle for spatiotemporal correlation of sensory signals from the environment. As statistical physics tells, each single correlation operation improves the sensitivity of intensity discrimination by a factor 2 . The earlier study gave arguments for the presence of seven such operations for first and second order correlation. These include the seeing of light, of movement and of edge. Each unit of seven neighboring hexagons contain a total of three mutually independent set of 7 twin pairs of receptor groups, two sets with the $R$ cones and one with the $G$ cones. Consequently, this processing system when in full binocular action improves the intensity discrimination of a single twin pair of receptor groups in one eye by a factor 128 , times 1.7 for the three subsets, times 1.4 for the two eyes or about 310 in total. Altogether, the retinal twofold spatiotemporal quantum coincidence mechanism is an inevitable expression of the general correlative signal processing in the nervous system (Reichardt 1961).

It is worth mentioning here further aspects of the distinctness of the system's time processing. Variation of frequency of a flickering light shows particularly at high intensity the abrupt disappearance of flicker at a distinct frequency around 50 cps. Similarly shows variation of the frequency of a black and white line grating, the rather abrupt invisibility of the lines at a distinct frequency that depends on eccentricity on the retina. Values around 30 cpd are quite common(Nes et al 1976). Also for moving targets beyond a critical speed that depends on retinal eccentricity, the visibility of movement disappears. For a moving point source, the perception of a line results (Kolers 1972). Also very remarkable is the fact that looking at the world is rather similar to the stroboscopic presentation of picture-sequences like in television 
and movies. In fact, this illusion is just complementary to the subjective stroboscopy as described by Schouten (Schouten 1967). This stroboscopy is also apparent in the wagon wheel illusion. In the latter, in daylight a sparked wheel of a forwards moving car seems under appropriate speeds to stay steady or even to turn in the wrong direction. The occurrence of the ghost beam next to the main scanning beam at the radar screen is another demonstration of vision's stroboscopy (Brink 1956). The attentive radar watcher observes two similar distinct bright moving beams that are 0.16 seconds apart. Increase of scanning speed results in proportionally increased distance. The illusion does not care on intensity of the beams. The two beams are the result of the second order correlation of light signals of a twin of receptor groups with the light signals of the neighboring twin groups. The existence of these phenomena is a rather definite confirmation of the eye's stroboscopic scanning of the environment. Effectively, human vision's perception of the environment is the perfect illusion of the real physics world. Under appropriate conditions, its illusionary nature appears like in TV, movies, Schouten's subjective stroboscopy, wagon wheel illusion, radar ghost beam, etc.

The remarkable spatial resolution in the vernier acuity remains a matter of serious concern for the proposals above. The ideal point spread function of the optics of the eye seems not to allow values for resolution below 30 to 40 seconds of arc. Because it concerns the visibility of distances between parallel lines, some averaging process might be involved, like in the second order correlation process for the perception of lines or movement (Bouman 2006). In this respect, it is required also to consider the firmly established knowledge of the involuntary movements of the eye, particularly the eye tremor with frequencies in the range around twenty cycles per second and amplitudes around fifteen arc seconds. Already this tremor seems not to allow the performance of vernier acuity, although directional effects of the eye movements in relation to direction of the lines in the stimulus may complicate 
the problem further. Riggs discovered that when by technical optical means the retinal image is stabilized against the tremor of the eye bulb, visual perception fades away (Ratliff and Riggs 1950). Consequently, the trembling of the image is essential for conscious perception and thus also for the achievements of vision in vernier acuity. Such extraordinary finding is for physics only conceivable when the time processing of the receptor signals is in pace with the synchronous tremor of both eyes (Ditchburn 1953). The main component of this tremor is in the horizontal plane. Thus, it concerns involuntary tremor of the fixation point either vice versa left/right or vice versa forwards/backwards. Because of the existence of the illusion of the Pulfrich pendulum and generally of the binocular fusion of retinal images of a moving environment, receptor signal processing also occurs in both eyes in accurate synchrony. This synchrony between the involuntary tremor and the time processing is similar to the set up of the image analysis in scanning microscopy. In both, the effective result is resolution that exceeds the limit set by diffraction of the optics of the system.

\section{Epilog}

These facts inevitable also imply that tremor of the bulbs and time processing of receptor signals have a common autonomous source. It is tempting to discuss this source further. A few considerations and established facts follow below. Some of them are speculative in nature. The skeletal musculature can show involuntary tremor, which is synchronous all over the body. This may include the tremor of the eye bulbs and of the middle ear bones. Dependence of auditory threshold on time of presentation is similar to this dependence for the visual threshold (Plomp and Bouman 1959). Vision's flicker fusion and the hearing's continuity effect show similar time dependences (Plomp 2001). The subject hears a ten to twenty times per second interrupted sound as continuous. In view of the existence of directional 
hearing, the processing of auditory stimuli in both ears occurs in accurate synchrony. Because of the fair sensitivity for changes in direction of the sound source, the accuracy of the time processing can reach the same value of less than half a millisecond as in binocular vision. The test subject can hear the simultaneity of a sound click for the ear and a light flash for the eye with precision of about a millisecond. A pair of vibrators at the biceps of both arms evokes the perception of a phantom vibration source outside the body (Bekesy 1960). The perception of its direction towards the corpus of the subject occurs rather precisely. This implies the synchrony of the time processing of tactile stimuli all over the body. The non-analog time component in visual pursuit movements and its correlation with distinct aspects of the alpha rhythm in the EEG both relate to the cortical control of the eye's fixation (Latour and Bouman 1961, Latour 1966). Therefore, the time processing of all sensory signals inclusive the proprioceptive signals of the motor system may be an autonomous synchronous affair. Its basic characteristics are rather definitely determined via experiments for vision converging in the pairing of successive time-quanta of 0.04 seconds. The site and nature of its autonomous source are unknown. Theoretical ad hoc studies demonstrated the possible generation of periodicities from the random action potentials of the resting activity of a set of interconnected neurons. They should describe the EEG's alpha rhythm but explain it not consistently. The time processing with time-quanta of the central nervous system needs a robust source. Can the action potentials of the myocardium's purkinje cells act as such? Either they make the heart's ventricle to contract or they themselves are the result of the ventricle's contraction. For generation of the time-quanta of the CNS, the latter possibility is the appropriate one. It seems absurd to pollute the integer description of experimental results above with such a seemingly absurd and unjustified idea. However, as a source they meet the required properties in an ideal way. Their frequency of occurrence is in the right range around twenty 
five times a second, their influence spreads by electric transmission via blood vessels, extracellular fluid and may be glia cells without any time delay all over the body. At cardiac arrest, any sensory and moving activity disappears and the subject becomes unconscious. The ECG leads only show an overall response of the potentials. The RC-type transmission from the blood vessels via the skin to the electrodes modifies this response. By their penetration through the skin, the electrodes of the EMG show in their grass like response the individual potentials of the purkinje cells at the myocardium. It would be a very intelligent design of a perfect scanning device that operates synchronic all over the sensory and motor systems of the body with accuracy far less than half a millisecond. The action potentials of the purkinje cells vary the tonus of the skeletal musculature, inclusive the muscles of the eye bulbs. This way the involuntary tremor of the eye's fixation is synchronous with the time processing of the light signals. This may all be reckless and unacceptable speculations. Particularly because of lack of reliable and expert knowledge on the way at which the transient mode of the purkinje action potential passes the membrane of the receptor or of proprioceptor of the motor system and makes the tonus of muscles change. 
In figure 4, a schematic picture of the human heart illustrates the site of the purkinje cells, their action potentials and a ECG lead.

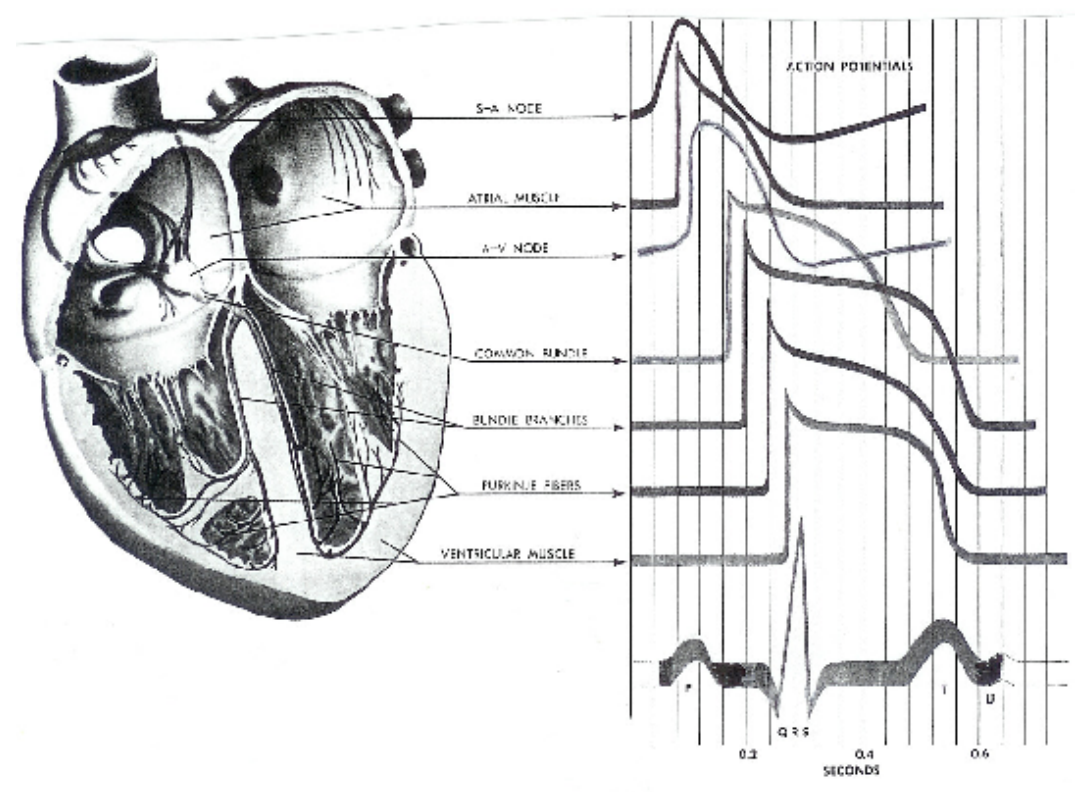

Figure 4

It is encouraging that the analysis of the signal processing of the visual system above naturally incorporates the existence of ON, OFF nerve elements, ON, and OFF activity of them. Every light, movement and edge signal carries one of two complementary polarities dependent on in which of the two receptor groups of a twin pair and in which of the two of a pair of time-quanta the first their constituting subliminal signals occurs. Each of the polarity-the ON as well as the OFF- has its own nerve net work. Elsewhere a more extensive analysis and description appeared(Bouman 2006).

The obvious reason for the distinct grouping and twinning of receptors are axonal functional connections in the nervous structure. However, there is not such a reason at hand for why a particular distinct period pairs with the preceding or the following period and is the first or the second of a successive pair of periods, neither for whether the pairings in both eyes and probably all over the central nervous system proceed conform. It is obvious that the actual 
pairing of time-quanta may change from the one into the other mode. Such a change results in the replacement of ON activity into OFF activity and vice versa. The consequence is the appearance of well-known rivalry phenomena, for instance a first convex appearing spheroid surface may turn into a concave one. It is still unclear what the actor is for the transient from the one to the other mode of pairing of time-quanta. They seem to occur rather at random, following a particular statistics (Levelt 1965). The synchronous tremors of the two eyes are in phase or in counter phase. For the first the binocular fixation point moves vice versa from left to right, for the second backwards to forwards. Likely, also the modes of tremor may change from the one into the other. For the rivalry phenomena is the relation between the modes of tremor of both eyes and the pairing mode of the time-quanta relevant. This is another point that naturally emerges from the analysis above and refers to the complicated aspects of these phenomena.

Trust in common sense, some understanding of sophisticated instrument design, specialized knowledge and experience in elementary statistical physics may suffice to consider the conclusions above.

\section{References}

Lecomte du Nouy, P. 1921. Energy and vision. J.Gen Physiol. 3,743-764. Bekesy, von G.1936. Uber die Hoerschwelle und Fuehlgrenze langsamer sinusformige Luftdruckschwankungen.Ann.Physik 26,554-556.

Bekesy, von G.1960. Experiments in hearing, Mc Graw Hill,New York.

Bouman,M.A. 1969.My image of the retina. Q.Rev.Biophys. 2, 25-64. Bouman,M.A. 2006. The simple perfection of quantum correlation in human vision.Progess in Neurobiology 78,38-60.

Bouman,M.A. and Brink, G. van de. 1952. On the integrate capacity in time and space of the human peripheral retina. J.Opt.Soc.Am. 42, 617-620 Bouman,M.A. and Walraven,P.L.1972. On threshold mechanisms for achromatic and chromatic vision. Acta Psychologica 36,176-189.

Brink,G.van de. 1967. Retinal summation and the visibilty of moving objects. Ph.D.Thesis, Utrecht University, The Netherlands.

Brink,G.van de. and Bouman,M.A. 1954.Variation of integrate capacity in 
time ansd space: an adaptational phenomea. J.Opt.Soc.Am. 44,616-620. Ditchburn,R.W. and Ginsborg,B.L. 1953. Involuntary eye movements during fixation. J.Physiol.119,1-12.

Kolers,P.A. 1972. Aspects of Motion Perception. Pergamon Press Ltd. Oxford,UK. Latour,P.L. and Bouman, M.A. 1961,A non-analog time component in visual pursuit movements. In Rosenblith, W.A. (Ed), Sensory Communication,MIT Press.

Latour, P.L.1966. Central control of eye movements. Ph D Thesis, Utrecht University, The Netherlands.

Levelt, W.J.M. 1965. On binocular rivalry. PhD Thesis, Leiden University.

Nes,F.L.van, Koenderink,J.J.,Nas,H., Bouman.M.A.1967. Spatiotemporal modulation transfer in the human eye.J.Opt.Soc.Am. 57,1082-1088.

Netter,F.H. 1972. The Heart. Ciba collection,Vol.5.

Mueller, C.J. 1954. A quantitave theory of visual excitation for the single photoreceptor. Proc.Natl. Acad.Sci. 10,853-863.

Plomp,R. and Bouman,M.A. 1957, The relation between hearing threshold and duration for tone pulses. J.Acoust. Soc.Am. 31,749-758.

Plomp,R. 2001, The intelligent ear. Lawrence Erlbaum Associates, Inc. Mahwah.NJ 04730. ISBN 0-8058-3867-8

Ratliff,F. and Riggs, L.A. 1950. Involuntary movements of the eye during monocular fixation. J.Exp.Psychol. 40,687-701.

Reichardt,W. 1961. Autocorrelation, a principle for the evaluation of sensory information by the central nervous system. In: Rosenblith, W.A.(Ed.) Sensory Communication. MIT Press, Canbridge,Mass.U.S.A. pp.303-319

Scholtes,A.M.W. and Bouman,M.A. 1977. Psychophysical experiments on spatial summation at threshold level of the human peripheral retina. Vision Res. 17,867-873.

Walraven,P. 1962.On the mechanism of clour vision.Ph.D.thesis,Utrecht University, The Netherlands. 
Figure 1

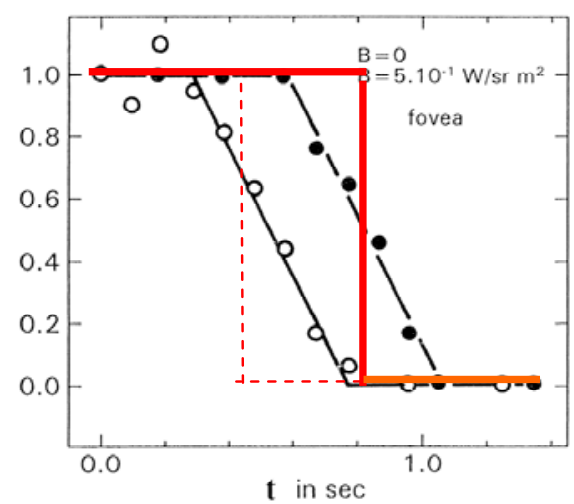

\section{$\mathbf{f}(\mathbf{t}, \mathbf{d})$}

Figure 1 shows in the red curves the probability of successful interaction of two subliminal responses in the fovea as a function of the time $t$ between them, for light of narrow spectral bandwidth around $500 \mathrm{~nm}$. The full drawn red curve applies for in the dark, the dashed curve for intense background. The black curves represent $f(t, d)$ in the dark $(B=0$, dots) and against an intense background $\left(B=5.10^{-1} \mathrm{~W} / \mathrm{sr}^{2}\right.$, open circles).

Figure 2

$(\mathbf{t}, \mathbf{d})$
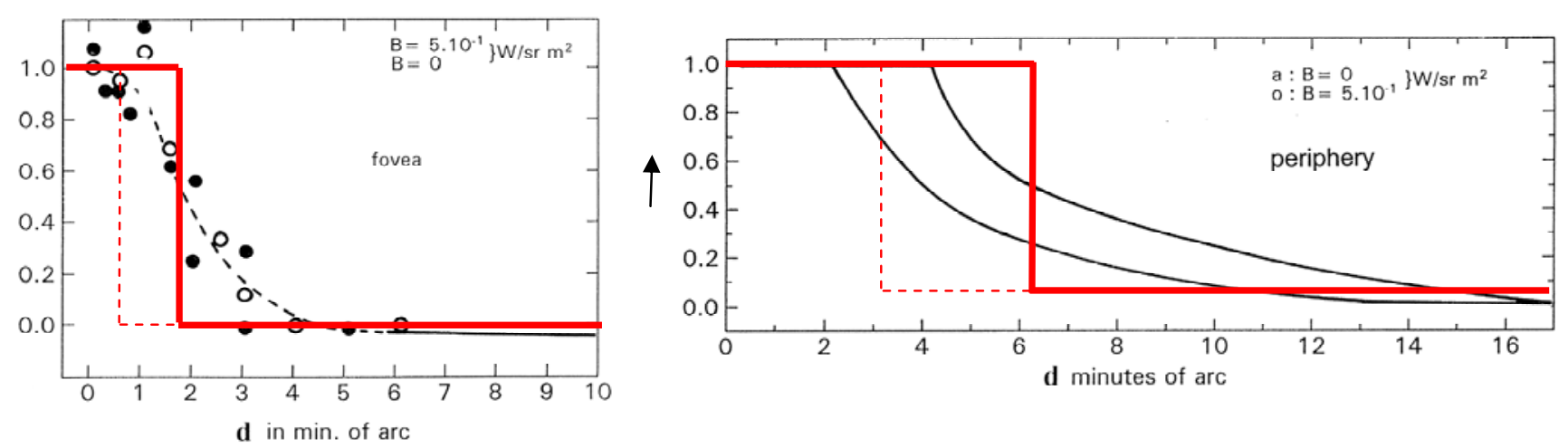

Figure 2 shows for the fovea for $500 \mathrm{~nm}$ light, the results of $f(t, d)$ as a function of $d$ in the dark (black open circles, $B=0$ ) and against background (black dots, $B=5.10^{-1} \mathrm{~W} / \mathrm{sr} \mathrm{m}^{2}$ ) The black dashed curve applies for any color of the light and intensity of the background, inclusive $B=0$. The red lines are for the probability of successful interaction of subliminal receptor responses in the dark (full drawn) and against intense background (dashed).

Figure 3 shows $f(t, d)$ in the full drawn black curves for seven degrees nasal from the fovea for $500 \mathrm{~nm}$ light, in the $\operatorname{dark}(B=0)$ and against intense background $\left(B=5.10^{-1} \mathrm{~W} / \mathrm{sr} \mathrm{m}^{2}\right)$. The red curves give the probability for successful interaction of subliminal responses in dependence of their distance in the retina, in the dark (full drawn) and against intense background (dashed). 


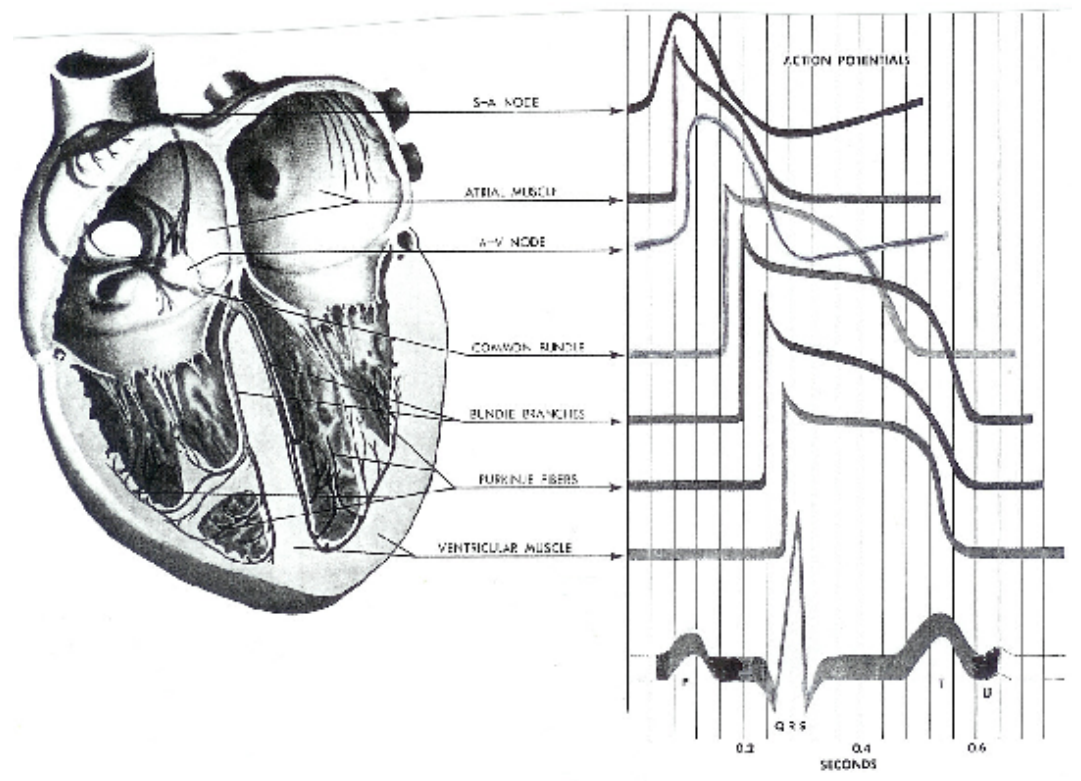

Figure 4 


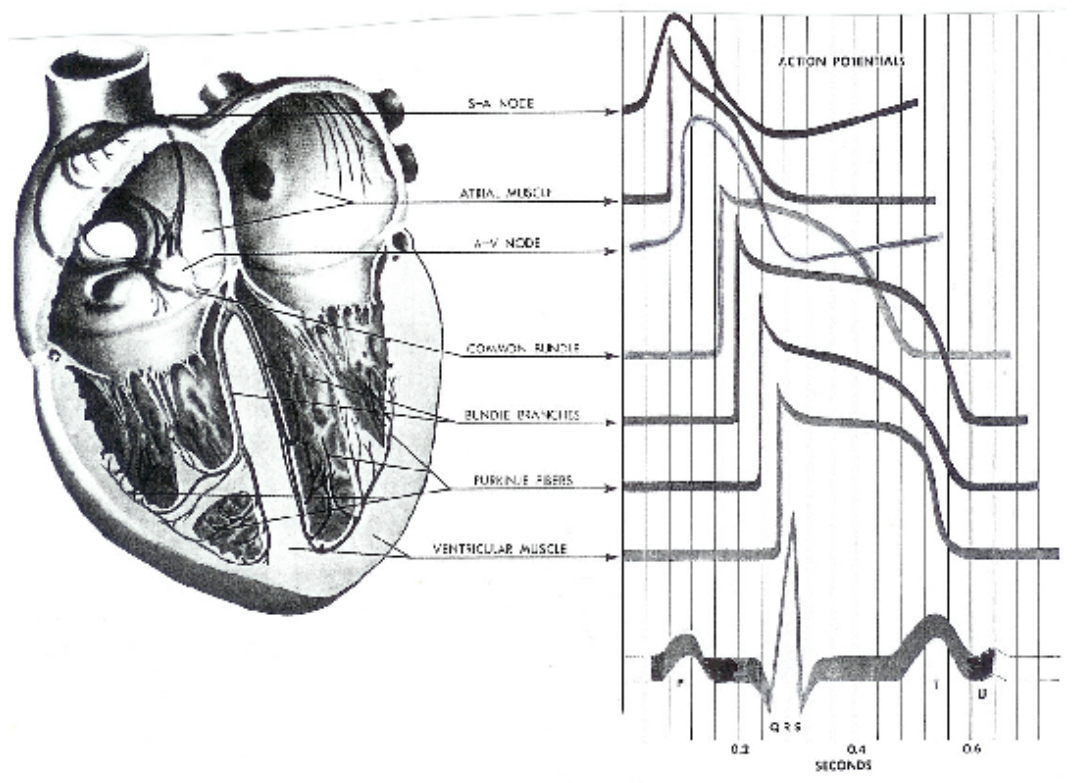

